\title{
INVESTIGACIÓN CUALITATIVA EN
} ADMINISTRACIÓN

\section{QUALITATIVE RESEARCH IN BUSINESS STUDIES}

Dr. Francisco López-Herrera (francisco_lopez_herrera@yahoo.com.mx) y Dr. Héctor Salas-Harms (hsalas@correo.fca.unam.mx). Facultad de Contaduría y Administración, Universidad Nacional Autónoma de México (México)

\section{Abstract}

This essay presents a general outlook and an analysis of the rationale behind the use of qualitative methods, including their being applied in research in Management. According to the views of methodological monism, the contribution of the conclusions which these methods yield, which are essentially subjective and not susceptible of being generalized, is considered too limited; while for advocates of constructivism and pluralism they are valuable in probing the depth of the phenomena of these disciplines. In fact, a lack of consensus prevails in these areas even in connection with what are qualitative methods and what are qualitative research techniques, associated to an indistinct use of these terms by some authors; which together with the number and diversity of qualitative methods makes it difficult to understand what actually is qualitative research.

Keywords: qualitative research, management, quantitative research, methods, techniques.

\section{Resumen}

Este ensayo presenta una panorámica general y un análisis de la visión que sustenta el empleo de los métodos cualitativos, incluyéndose su aplicación a la investigación en Administración. Bajo los planteamientos de un monismo metodológico, la aportación de conclusiones no generalizables y de subjetividad inherente de tales métodos se considera limitada; mientras que para los adherentes al constructivismo y al pluralismo, es de gran valía en la exploración de la profundidad de los fenómenos de estas disciplinas. En sí, prevalece en el medio una falta de consensos incluso con respecto a qué son los métodos cualitativos y qué son las técnicas cualitativas de investigación, derivada del uso indistinto que dan algunos autores a esos términos; lo que aunado a la cantidad y diversidad de métodos cualitativos crea dificultades para comprender qué es en realidad la investigación cualitativa.

Palabras clave: investigación cualitativa, administración, investigación cuantitativa, métodos, técnicas. 


\section{Introducción}

Primeramente, para situar en un contexto general las particularidades de los métodos cualitativos, así como sus coincidencias y divergencias con los métodos cuantitativos, en la primera sección de este ensayo se enmarcan ambos tipos de investigación en el ámbito de la literatura que comprende las diversas clases de estudios sobre Administración; distinguiéndose, por una parte, entre aquéllos de carácter propositivo y, por la otra, los estudios propiamente de investigación, ya sean de índole descriptiva, cuando emplean métodos cualitativos, o de índole explicativa, basados en métodos cuantitativos (1).

Subsecuentemente, y antes de adentrarse en la discusión sobre los métodos cualitativos, en la sección 2 se precisa inicialmente qué son los métodos y qué son las técnicas de investigación, dilucidándose brevemente el significado de ambos términos; particularmente porque en ocasiones se emplean como sinónimos. Asimismo, se clarifica en qué consiste la investigación cualitativa; tarea que no es fácil pues no existe un origen único de tales métodos, sino que éstos son más bien un enfoque de investigación que ha surgido en paralelo en diversas disciplinas sociales. Finalmente, se presentan las principales diferencias entre la investigación cualitativa y la de tipo cuantitativo, útiles para delimitar a la investigación cualitativa, destacando la relación entre la investigación cualitativa y la investigación en Administración.

Una idea clara de la naturaleza y las características esenciales y comunes de los métodos de la investigación de tipo cualitativo requiere comprender qué es la investigación cualitativa y qué propósitos persigue. También resulta conveniente contar con una panorámica de los métodos comúnmente empleados en ese tipo de investigación. En la sección 3 de este ensayo se ofrece una perspectiva general de cómo aborda la investigación cualitativa las tareas de investigación y de las características generales de sus principales métodos surgidos en distintas disciplinas sociales. Se inicia presentando los objetivos de la investigación cualitativa y las preguntas específicas de ese tipo de investigación. Se explica también por qué es difícil hacer un recuento exhaustivo de los métodos cualitativos de investigación, presentando como ejemplos algunos de los más conocidos.

Es de interés particular ver cómo perciben el uso de métodos cualitativos en la investigación en Administración quienes de alguna manera están involucrados en ella. Por tal motivo, en la sección 4 se analizan los resultados de la investigación de Cassel et al (2005) que ofrecen una perspectiva general de cómo se percibe la investigación cualitativa y qué se piensa de la aplicación de sus métodos a la investigación en Administración, destacándose tanto los argumentos a favor de su empleo, como aquéllos de quienes los consideran útiles sólo en las primeras etapas de una investigación, pero muy limitados en sí mismos. Por último, en la siguiente sección se presentan algunas reflexiones y comentarios finales.

\section{Los Estudios sobre Administración}

Los estudios sobre Administración pueden dividirse en aquéllos que tienen por finalidad el diseño de herramientas para su aplicación en la práctica profesional en las organizaciones, por una parte; y por la otra, en aquéllos que tienen por objeto analizar el comportamiento de las mismas, en general, así como el impacto que produce en su desempeño la instrumentación de cada una de dichas herramientas administrativas, en particular. Los estudios del primer tipo son de carácter propositivo; mientras que los que se abocan a investigar comportamiento y desempeño de las organizaciones, dependiendo de los métodos de investigación que empleen, se subdividen a su vez en descriptivos y explicativos, cuando aplican métodos cualitativos o cuantitativos, respectivamente (Véase el cuadro 1). 
Cuadro 1. Estudios sobre Administración

\begin{tabular}{|c|c|c|}
\hline Tipo & Finalidad & Productos \\
\hline Propositivos & $\begin{array}{c}\text { Generar herramientas para la práctica } \\
\text { profesional en las organizaciones }\end{array}$ & $\begin{array}{c}\text { Estrategias, modelos, técnicas, } \\
\text { procedimientos, sistemas, etc. }\end{array}$ \\
\hline \multirow{2}{*}{ Descriptivos } & $\begin{array}{r}\text { Investigar el comportamiento y desempeño } \\
\text { de las organizaciones }\end{array}$ & Estudios de caso cualitativos \\
\cline { 2 - 3 } Explicativos & & Artículos con análisis cuantitativo \\
\hline
\end{tabular}

Fuente: Elaboración propia.

Sin pretender referir estas ideas al ámbito de la controversia sobre la cientificidad de la Administración -o en general, sobre la cientificidad de las disciplinas sociales- puede hacerse una analogía con las dimensiones que corresponden a ciencia y tecnología. La ciencia se ocupa de lo generalizable a partir de lo singular, y busca discernir en los fenómenos de la naturaleza leyes de aplicación universal que hablen de la uniformidad o regularidades en el curso de la misma naturaleza, y que revisten un aparente carácter normativo (Berkeley 1910:103, Kolakowski 1981:315, Waters 1998:5, Ordóñez 2003:11 y 59).

Por su parte, la tecnología -en referencia a la tecnología de procesos, no de productos- que a partir de la tercera revolución industrial se deriva crecientemente en forma directa del conocimiento científico, como parte de un continuo de actividades (ciencia básica-ciencia aplicada-tecnología-ingeniería) que paulatinamente se han integrado sobre todo al interior de las organizaciones productivas, es entendida como la producción de herramientas y el aprovechamiento de habilidades humanas para alcanzar fines prácticos (Lussier 1997: 585-6, Ordóñez 2003: 59 y 11.)

En estos términos, continuando con dicha analogía, los estudios descriptivos y explicativos se ubicarían en la esfera del conocimiento de corte científico; y los propositivos se encontrarían en el contexto de la producción de tecnología. En sí, los métodos cualitativos de investigación surgen en las disciplinas sociales en respuesta a las limitaciones que se disciernen en los métodos cuantitativos asociados con el positivismo, que se estiman incapaces de abordar la complejidad de los fenómenos sociales. Pero estrictamente, el carácter meramente casuístico de los estudios descriptivos bajo métodos cualitativos, cuyo propósito es buscar establecer conclusiones no generalizables, los colocaría realmente en una etapa pre-científica o clínica, en la cual el análisis de un número importante de estudios de caso a profundidad sobre un fenómeno específico permite al investigador comenzar a encontrar patrones y discernir regularidades con relación a la misma, para subsecuentemente formular hipótesis sobre las relaciones causales observadas, que serán sometidas finalmente a corroboración mediante análisis cuantitativo con rigor metodológico en la etapa propiamente científica.

El conocimiento obtenido a partir de establecer con altos niveles de confianza estadística tales regularidades observadas en el comportamiento de las organizaciones y su administración, jugará posteriormente un papel determinante al ser aplicado en el análisis para la toma de decisiones. Esto es así cuando se ofrece al tomador de decisiones en una empresa evidencia empírica sólida, p. e., de que una de las estrategias de crecimiento produce consistentemente un desempeño financiero y de mercado superiores al de las 
estrategias alternativas; o de que los costos unitarios de operación disminuyen conforme aumenta la escala misma de operación de las compañías que pertenecen a ciertos giros (Rumelt 1986, Merino 1985).

Esto es así, igualmente, cuando un ejecutivo de una compañía puede acceder a resultados de investigación, que han sido validados al replicar los estudios originales así como al relajar los supuestos y modificar las metodologías iniciales, y que muestran, p. e., que de las tres técnicas de valuación de acciones de uso más común, una de ellas regularmente sobre-valúa y otra regularmente sub-valúa las valuaciones obtenidas mediante la técnica de aplicación más frecuente en el medio; o de que variables financieras tales como las de rentabilidad, eficiencia, valor y otras, siguen patrones de co-evolución, cuyas etapas coinciden con las diferentes etapas del modelo del ciclo de vida de la industria; entre muchos otros ejemplos más (Saavedra 2002, Gómez 2004).

Por su parte, la experiencia incorporada en las herramientas que constituyen el conjunto de tecnologías administrativas, que representan esquemas bajo los cuales ordenar recursos, actividades u operaciones para alcanzar objetivos o resultados determinados, o para transformar en productos o servicios diversas clases de insumos propios de la gestión de las organizaciones, permiten a sus directivos optimizar eficacia, eficiencia y calidad en la conducción de éstas últimas, al implantar hacia su interior dichos elementos tecnológicos. Esto es así, p. e., al definir y comunicar la visión de una organización, como una herramienta para desarrollar e integrar los elementos que permitan capturar el espíritu y energía de la misma y guiarla hacia su futuro más deseable (Jick 1993:83-97, Lussier 1997:139-41).

Asimismo, esto es así, p. e., al plantear e instrumentar estrategias, fijando objetivos y metas de largo plazo, trazando cursos alternativos de acción y asignando distintos tipos de recursos para alcanzar aquéllos; al establecer y aplicar modelos, como representaciones simplificadas de la realidad, para el análisis de situaciones en las tareas de planeación y toma de decisiones; o al rediseñar a una organización bajo una visión de sistemas, enfocándose a conceptualizarla y dirigirla como un todo en el cual diversos elementos mutuamente relacionados se articulan e interactúan desde una perspectiva holística del proceso administrativo para encauzarla a cumplir su misión y modelarla a imagen de la visión delineada (Jick 1993:401-12, Lussier 1997:104-21, 40-4 y 22, Hill y Jones 1995:5-7 y 39-43).

\section{Métodos y técnicas de investigación cualitativa e investigación en Administración}

Los métodos y las técnicas de investigación cualitativa

A veces se utilizan los términos "método(s) de investigación" y "técnica(s) de investigación" como sinónimos; y muy frecuentemente se encuentra que algunos autores se refieren a métodos y técnicas sin establecer una frontera clara entre unos y otras. Tal uso indiscriminado naturalmente complica el panorama cuando se trata de distinguir entre cuáles son los métodos y cuáles son las técnicas, que no significan exactamente lo mismo, a pesar de su íntima vinculación. Una explicación de la falta de consenso sobre cuáles son los métodos y cuáles son las técnicas podría ser que la investigación cualitativa exige reconocer múltiples realidades en su afán de capturar la perspectiva de los miembros del grupo social investigado. Otra fuente de la ausencia de consenso para una definición que permita separar los métodos de las técnicas es la estrecha relación entre diseño, técnicas, análisis y construcción teórica, señalada por Scribano (2000). Aunque podría parecer trivial, aclarar qué se debe entender por método o métodos y qué por técnicas en realidad es importante pues, señala este autor, no reconocer sus diferencias puede llevar a confusiones sobre lo que realmente es la investigación cualitativa. 
Aunque ineludiblemente relacionados en el quehacer de la investigación, estos términos no significan lo mismo ni son sustitutos intercambiables, y en última instancia se refieren a aspectos precisos de la investigación claramente diferenciados. Para Grawitz (1974) el método puede entenderse en cuatro sentidos fundamentales. En el sentido filosófico, en el nivel más alto de abstracción, el método denota los procedimientos lógicos que acompañan a toda investigación científica y que debe seguir el investigador para acercarse a la verdad y verificarla. El método también se refiere a la actitud concreta o posición que el investigador adopta frente al objeto de estudio, por lo que en este nivel el método orienta la forma en que se organiza y lleva a cabo la investigación. Un tercer sentido que adquiere el método es por su vinculación con un intento de explicación, por lo que la posición filosófica seleccionada por el investigador influye en el método a seguir en la investigación. Por último, el método puede considerarse como algo propio del ámbito particular de la investigación, es decir, el método se concibe entonces como acorde con el campo de conocimiento del investigador.

Para Alonso (1996), en cualquiera de los niveles a que se haga referencia no hay algo común entre el significado de método o métodos y el de las técnicas, a las que considera como: "los procedimientos operativos, rigurosos, bien definidos, transmisibles y susceptibles de ser aplicados repetidas veces en las mismas condiciones". Claro que la diferencia de significados no implica inexistencia de vínculos estrechos entre métodos y técnicas de investigación; lazos aún más ostensibles en el transcurso de la investigación. Alonso señala que la elección de las técnicas depende del objetivo buscado y del método de trabajo.

La investigación cualitativa y la investigación en Administración

Según Ragin, Nagel y White (2004), en las disciplinas sociales existe una aparente división tajante entre la investigación cualitativa y la investigación cuantitativa. Para Pita y Pértegas (2002), la investigación cualitativa se caracteriza por evitar la cuantificación y basarse en descripciones narrativas. Los investigadores que optan por esta vía, realizan su investigación mediante registros narrativos de los fenómenos que estudian, valiéndose de técnicas no cuantitativas como, por ejemplo, la observación participante y entrevistas no estructuradas.

La diferencia fundamental entre ambos tipos de investigación, es que la cuantitativa estudia la asociación o relación entre variables cuantificadas, en tanto que la cualitativa lo hace en contextos estructurales y situacionales. La investigación cualitativa trata de identificar la naturaleza profunda de las realidades, su sistema de relaciones, su estructura dinámica; la investigación cuantitativa trata de determinar la fuerza de asociación o correlación entre variables, buscando la generalización de los resultados alcanzados a través del estudio de una muestra para hacer inferencia sobre una población.

Para Ragin, Nagel y White (2004), más que una dicotomía entre investigación cualitativa e investigación cuantitativa, se trata en realidad de un continuo en el que se pueden identificar dos extremos claramente definidos. En uno de esos extremos se encuentra la investigación de tipo cuantitativo, caracterizada por suponer que existe objetividad en el análisis de poblaciones, casos y variables supuestamente definidos de manera clara, suponiendo también que las teorías e hipótesis pertinentes están bien especificadas. En el extremo opuesto del continuo se encuentra la investigación cualitativa que descarta esos conceptos, considerando además que la teoría convencional es sospechosa y que en el proceso de la investigación se desvanece la distinción entre el investigador y el objeto de estudio. Esos autores reconocen que entre los dos extremos se encuentran muchas estrategias de investigación híbridas que combinan tanto métodos cualitativos como cuantitativos para llevar a cabo el análisis y las inferencias pertinentes. 
La investigación cualitativa ha cobrado popularidad a raíz de los cambios paradigmáticos que se experimentan en el quehacer de las disciplinas sociales, sin embargo, no es del todo nueva. Según González (2000) existen antecedentes de este tipo de investigación desde finales del siglo XIX en el campo sociológico. Entre 1910 y 1940 "La Escuela de Chicago", importante corriente sociológica estadounidense, hizo un uso amplio de sus métodos (Cereceres 2003). No obstante, en los años cincuenta del siglo pasado se perdió el interés en esos métodos, cediendo espacio ante el predominio de los métodos cuantitativos. En la década siguiente resurgió el interés por los métodos cualitativos. Actualmente la investigación cualitativa se emplea por investigadores de diferentes disciplinas sociales y por profesionales de diversos campos, interesados en asuntos relacionados con el comportamiento y el funcionamiento humanos, por lo que esta modalidad de investigación se utiliza ampliamente para estudiar organizaciones, grupos o individuos. La cercanía de la teoría administrativa a las disciplinas sociales hace comprensible y totalmente natural que en la investigación en Administración exista interés por el uso de los métodos cualitativos.

Gephart (1999) señala que entre los años setenta y ochenta del siglo XX surgieron importantes preocupaciones respecto de las limitaciones de los datos y métodos cuantitativos asociados con el paradigma positivista, prevaleciente en ese entonces en la investigación realizada en el terreno de las disciplinas sociales como herencia de las disciplinas naturales. De acuerdo con Gephart, el paradigma positivista supone que el mundo es objetivo y que los métodos científicos pueden representar y medir con medios adecuados la realidad observable, por lo que dicho paradigma busca explicar y predecir las relaciones entre las variables clave. Para algunos críticos del enfoque positivista, como Guba y Lincoln (1994), los métodos de cuantificación que siguen los investigadores de corte positivista a menudo se olvidan de aspectos importantes que se encuentran dentro del contexto de la investigación. En particular, señalan que las medidas cuantitativas imponen significados e interpretaciones externas a los datos, excluyendo de esta forma significados e interpretaciones que los miembros del grupo social estudiado atribuyen a la realidad observada.

Los defensores de la investigación cualitativa sostienen que las aproximaciones cualitativas permiten una visión del mundo más amplia que los enfoques cuantitativos ya que los datos no cuantificables pueden ofrecer una visión holística al basarse en experiencias y actitudes de los miembros del grupo social estudiado, permitiendo que se deriven conocimientos y explicaciones causales (Pedone 2000). Martínez (1999) y Ferrarotti (1993) sostienen que los métodos de la investigación cualitativa buscan la comprensión holística de una totalidad social dada. Es decir, la investigación cualitativa pretende alcanzar la comprensión de las relaciones complejas entre todo lo que existe en torno a la realidad estudiada (Stake 1999). De esta forma, los métodos cualitativos constituyen para muchos investigadores de las disciplinas sociales una línea natural del desarrollo de la investigación en sus áreas y recurren a ellos tratando de entender la naturaleza profunda de las realidades socio-culturales y sus estructuras dinámicas, esperando que puedan dar razón de los comportamientos y manifestaciones de los grupos humanos.

Al reconocer que las relaciones que surgen en las organizaciones son complejas, no sorprende que a los métodos cualitativos se les dé un lugar destacado en las investigaciones sobre las organizaciones por los estudiosos de la Administración. A fin de cuentas, la teoría administrativa ha recibido contribuciones importantes como resultado de investigación cualitativa. Ejemplo específico de esas contribuciones son las aportaciones que fundamentaron la denominada corriente de las relaciones humanas en el pensamiento administrativo. La base para el planteamiento teórico de esa escuela del pensamiento administrativo se derivó de los estudios hechos por Elton Mayo y a un grupo de investigadores en la Western Electric de 
finales de los años veinte a principios de los treinta del siglo XX. Parte importante de los descubrimientos hechos por Mayo, director de la investigación, y Roethlisberg, su ayudante principal, se basó en entrevistas al personal obrero por aproximadamente doce años. Otro ejemplo más reciente de investigación en Administración basada en métodos cualitativos es el estudio de Henry Mintzberg, quien a partir de sus observaciones de campo planteó una teoría de las funciones del administrador con base en los papeles que éste lleva a cabo en sus tareas cotidianas.

Otras áreas de estudios sobre las organizaciones también se caracterizan por el empleo de métodos cualitativos para llevar a cabo sus investigaciones. Por ejemplo, Rendón y Montaño (2004:103-4) señalan que las corrientes del análisis organizacional, del análisis institucional, la sociología de las organizaciones y la sociología de la empresa se caracterizan por la investigación con métodos cualitativos. Afirman esos autores que la corriente de los estudios organizacionales adquiere una creciente orientación a los métodos cualitativos y que tanto la teoría de la organización como la sociología del trabajo emplean métodos cualitativos, además de los cuantitativos.

\section{Panorámica de la investigación cualitativa y sus métodos}

En principio podría pensarse que la investigación cualitativa es simplemente aquella que no ofrece un análisis estadístico o algún tipo de cuantificación de los datos analizados. Sin embargo, aunque la ausencia de técnicas cuantitativas puede ser un rasgo distintivo de la investigación cualitativa, no es suficiente para describirla como un modelo de investigación científica. De acuerdo con el paradigma de la investigación cualitativa, el principal interés del investigador es entender y describir una escena social y cultural desde adentro. En términos generales, la investigación de corte cualitativa busca responder a las preguntas sobre el por qué se comportan las personas como lo hacen, cómo se forman sus opiniones y actitudes, cómo se ven afectadas por su entorno, cómo y por qué se han desarrollado las culturas en la forma en que lo han hecho; asimismo, trata de explicar las diferencias observables entre los grupos sociales (Hancock 2002). Los problemas de investigación que se plantean los estudiosos de las disciplinas sociales adeptos al modelo cualitativo son las formas en las que los individuos y los grupos sociales de interés constituyen e interpretan las organizaciones y las sociedades, es decir, cómo constituyen los significados (Gephart 1993). Según Schwartzman (1993), la investigación cualitativa facilita el aprendizaje de culturas y estructuras organizacionales porque brinda al investigador formas de examinar el conocimiento, el comportamiento y los artefactos que los participantes comparten y usan para interpretar sus experiencias. En términos generales, se podría decir que la investigación cualitativa busca responder preguntas como: ¿por qué?, ¿cómo? y ¿en qué forma?; diferenciándose de las preguntas que se plantea la investigación de tipo cuantitativo tales como: ¿cuánto?, ¿cuántos?, ¿con qué frecuencia? y ¿en qué medida? Para Taylor y Bogdan (1987), como consecuencia los métodos cualitativos producen datos descriptivos.

De acuerdo con Ragin, Nagel y White (2004), la investigación cualitativa se caracteriza por el estudio a profundidad de un número pequeño de casos, incluyendo el estudio de caso único, entendiéndose por casos acciones, eventos, narraciones, instituciones, organizaciones, y otras categorías sociales como ocupaciones, países y culturas, por ejemplo. Además, afirman, la investigación cualitativa busca conocimiento detallado de casos específicos, tratando de descubrir cómo ocurren (u ocurrieron) las cosas. Agregan que la meta primaria es que los hechos sean comprensibles, con menor énfasis en la extracción de inferencias o de predicción a partir de los patrones que emergen al comparar diferentes casos. Por lo general, los estudios cualitativos son investigaciones a muy pequeña escala pero intensivas por la profundidad con que tratan de 
explorar la experiencia cotidiana de la gente y sus comunidades en diferentes tiempos y espacios. En el enfoque cualitativo, tanto la posición que adopta el investigador, así como sus experiencias, perspectivas y prejuicios son significativos en el desarrollo y los resultados de la investigación (Philip 1998).

Algunos estudiosos consideran que dada la amplitud y profundidad que pretende, la investigación cualitativa exige reconocer múltiples realidades para abordar el objeto de estudio al mismo tiempo que trata de capturar la perspectiva del sujeto investigado. La documentación de múltiples perspectivas de la realidad es crucial para entender por qué la gente piensa y actúa de diferentes formas (Fetterman 1989). Bajo la perspectiva de una disciplina social capaz de abordar y comprender la realidad social analizada desde una perspectiva amplia y múltiple, la investigación correspondiente debe caracterizarse por ser transdisciplinaria en lugar de mono o multidisciplinaria y más bien heterogénea que homogénea. Como resultado de la multiplicidad de enfoques no hay definición única de los métodos de investigación cualitativos, por ejemplo, González (2000:2) sostiene que algunos consideran a la investigación cualitativa como una forma de hacer ciencia diferente al enfoque positivista; otros identifican lo cualitativo en un nivel instrumental sin apartarse de la epistemología positivista.

Scribano (2000) señala que si bien los enfoques cualitativos de la investigación en las disciplinas sociales se caracterizan por la íntima relación existente entre diseño, técnicas, análisis y construcción teórica, que en la práctica invalida una separación tajante de las etapas de la misma, es lícito y además conveniente intentar reagrupar dichas prácticas según alguna orientación dominante que las asemeje y diferencie. Gephart (1999) clasifica los métodos de investigación en las disciplinas sociales agrupándolos de acuerdo con tres paradigmas epistemológicos prominentes: el positivismo, el enfoque interpretativo y el postmodernismo crítico. Para el paradigma positivista, la realidad es objetiva y susceptible de analizarse en términos de relaciones y asociaciones existentes entre las variables y hechos, según lo establece la teoría considerada pertinente. Por ello, se puede decir que la unidad de análisis en la investigación positivista es la variable. De esta forma, sostiene Gephart, en el terreno de la investigación en Administración el investigador se centra en el estudio de variables organizacionales y del contexto que producen las acciones observables en la organización. La teoría de las contingencias y la teoría de sistemas se encuentran entre las teorías más representativas del enfoque positivista en el desarrollo teórico de la Administración.

Según Schwandt (1994), la investigación interpretativa se ocupa fundamentalmente de los significados que los miembros del grupo social atribuyen a las situaciones en que se encuentran, por lo que ese enfoque de investigación busca en primera instancia encontrar los patrones existentes. Se aparta del positivismo pues supone que el conocimiento y los significados son actos de interpretación, razón por la cual no existe conocimiento objetivo en forma independiente del pensamiento y del razonamiento humanos. Aunque existen diversas corrientes asociadas con el paradigma interpretativo, como lo señala Gephart (1999), una característica común a todas ellas es que su principal interés son los significados subjetivos, es decir, cómo aprenden, entienden y se explican los eventos y escenarios sociales en los que se encuentran inmersos los miembros de la sociedad. Por ello, los adeptos a este enfoque buscan describir y entender esos significados, siendo sus unidades de análisis precisamente el significado y el acto simbólico.

El post modernismo crítico es producto de la combinación de dos diferentes visiones del mundo: la teoría crítica y la escuela post moderna. La teoría crítica es una corriente que surge de la filosofía y del pensamiento político alemán basados en la herencia hegeliana, marxista, kantiana y weberiana. Aunque los seguidores de esta corriente se apartan del marxismo ortodoxo en muchos aspectos fundamentales, 
mantienen como centro de atención la naturaleza cambiante del capitalismo y las formas de dominación, injusticia y subyugación producidas por esta forma de organización socioeconómica. La llamada escuela post moderna surge en parte del trabajo de pensadores franceses como Lyotard, Derrida y Foucault, quienes conocían muy bien al pensamiento crítico pero consideraban que éste estaba limitado en relación con los cambios en la naturaleza y la lógica del capitalismo. Características básicas del pensamiento de esta escuela son el abandono de narrativas de amplio alcance, centrándose en un enfoque más limitado, y el reconocimiento de la naturaleza cambiante de las condiciones sociales que dificulta el desarrollar y sostener la reflexión crítica. Según Gephart (1999), algunos investigadores de esta corriente usan también métodos convencionalmente asociados con el positivismo, como es el caso de la investigación basada en encuestas.

Ragin, Nagel y White (2004) señalan que la investigación cualitativa puede servir para probar si los mecanismos no observables que propone una teoría determinan la relación entre ciertas variables. Asimismo, sostienen esos autores, la investigación cualitativa puede ser también útil para investigar los casos anómalos, aquellos que la teoría no puede explicar, así como para abordar el estudio de fenómenos para los que no existe una teoría o, si existe, ésta es insuficiente. Por otra parte, la credibilidad de los resultados de la investigación cualitativa también sirve como parámetro para evaluar la calidad de dichos resultados (Gephart 1999, Ragin, Nagel y White 2004). Por otra parte es necesario reconocer que muchos investigadores consideran que es conveniente combinar la investigación cualitativa con la cuantitativa. Un ejemplo de tal combinación sería el uso de métodos cualitativos para obtener información respecto a los significados, sentimientos y cultura existentes en un grupo social, en tanto que la investigación cuantitativa podría servir para medir características de tipo estructural, contextual e institucional. Asimismo, la investigación cualitativa podría ofrecer tipologías útiles como categorías en el análisis cuantitativo.

Según Bericat (1998), de la integración de métodos cualitativos y métodos cuantitativos en la investigación pueden surgir tres tipos de estrategias a seguir: complementación, combinación y triangulación. Mediante la complementación se obtienen dos imágenes del proceso o fenómeno estudiado, una cualitativa y una cuantitativa, sin que predomine alguna de ellas en el análisis. La estrategia de combinación se diferencia de la complementación porque existe el predominio claro de una imagen sobre la otra. Por su parte, la triangulación supone la confrontación de ambas perspectivas, es decir, no se trata de analizar diferentes aspectos del fenómeno o situación bajo estudio, sino de reconocer el mismo aspecto a partir de dicha confrontación. Según Vera (2005) y Vera y Villalón (2005), la estrategia de triangulación puede llevarse a cabo en varios niveles. En primer lugar se pueden confrontar la congruencia (o incongruencia) entre los datos obtenidos mediante los métodos cuantitativos y los obtenidos a través de los métodos cualitativos. Otro nivel de triangulación es cuando ésta se hace al confrontar los resultados con las bases teóricas que sustentan la investigación de corte cuantitativo con las correspondientes a la de tipo cualitativo. Asimismo, la triangulación puede hacerse en el nivel metodológico o, incluso, confrontando los resultados obtenidos mediante la aplicación de métodos cualitativos con los que han alcanzado otros investigadores que han seguido métodos cuantitativos. Existiendo también la triangulación múltiple que implica llevar a cabo dos o más de las triangulaciones enunciadas.

De acuerdo con lo anterior, es necesario reconocer que los enfoques y objetivos de la investigación cualitativa pueden ser de naturaleza tan diversa entre sí, como lo es la naturaleza de las disciplinas sociales en cuyo seno surgieron los métodos cualitativos (Valles 1997). Siendo sus orígenes tan diversos y heterogéneos, tanto histórica como social y conceptualmente, las diversas orientaciones teóricas presentes en cada una de las disciplinas sociales han producido una variedad de perspectivas teórico metodológicas 
(Valles 1997). La multiplicidad de métodos y la amplitud de su uso hacen que, por una parte, resulte prácticamente imposible ofrecer una lista exhaustiva de ellos, pues se puede decir que la investigación en cada una de las disciplinas sociales cuenta con sus métodos preferidos

Para tener una idea general de las características esenciales y del origen plural de los métodos cualitativos, pueden citarse el método etnográfico, el análisis conversacional, el análisis del discurso, la investigaciónacción participante y la grounded theory, como ejemplos de dichos métodos.

Método etnográfico. La etnografía consistente en el estudio de las culturas y las personas. Surge en la antropología, extendiéndose su método de estudio en la actualidad a otras disciplinas sociales, como la sociología por ejemplo. Quienes usan este método buscan formarse una imagen realista del grupo estudiado, de forma tal que se pueda contribuir a la comprensión de grupos u organizaciones más amplias pero que compartan características similares (Bernal 2000). Por lo general, el investigador que sigue el método etnográfico no formula previamente los problemas de investigación, éstos van presentándose en el transcurso del estudio. Por lo que el investigador trata de interpretar los datos, obtenidos mediante técnicas como las entrevistas y la observación participante, desde la perspectiva de la población bajo estudio. Los resultados se expresan como si fuesen expresados por los sujetos mismos.

Análisis conversacional. Este método, surgido en la sociología, estudia procesos psicosociales que se dan en situación de interacción personal. La idea fundamental es que el lenguaje puede comprenderse por su uso y, por ello, reconoce la conversación como la forma social del lenguaje. También supone que el significado no es el resultado de una operación llevada a cabo por el investigador que observa, sino más bien surge del significado que las personas que se encuentran en una situación específica atribuyen a sus propias acciones, a la propia situación y a las consecuencias derivadas de esas acciones. Por lo anterior, de acuerdo con la posición adoptada por los investigadores para entender cómo construyen las personas los significados, los procesos bajo estudio son construidos por las personas que interactúan en ellos y los significados los proporcionan dichas personas, no quien hace el análisis. Asimismo, según este método, el papel de los investigadores no es comprender qué ocurre ni cuál es el significado de lo que está ocurriendo, sino más bien lo que se debe entender es qué significado le atribuyen a sus acciones los participantes de la situación concreta bajo estudio.

Análisis del discurso. De manera general puede decirse que el análisis del discurso trata de realizar inferencias valiéndose de la identificación sistemática y objetiva de las características de especificidad del texto (sea escrito u oral). Las raíces y fundamentos teóricos de este método corresponden a la lingüística, pero se ha extendido también su uso a la investigación en psicología social. Desde la perspectiva lingüística, algunos estudiosos entienden el discurso como el lenguaje en uso, incluyendo la comunicación entre actores en un contexto determinado. Otros lingüistas conciben al discurso no sólo como un fenómeno social, sino también reconocen en él un carácter ideológico y un carácter dialógico. Un tercer grupo de lingüistas enfatizan la conceptualización del discurso como una práctica social, ideológica y de significación que construye y reconstruye las entidades sociales. En la psicología social la concepción del discurso y del análisis del discurso se ha orientado psicosocialmente, estudiando el papel del lenguaje y de la interpretación en los procesos sociales.

Investigación acción participativa. Este método surge de la sociología y considera que las personas construyen la realidad en la que viven. Sostiene que los grupos y las comunidades en las que están insertos tienen su propio desarrollo histórico y cultural, es decir, antecede y continúa a la investigación. La relación entre el investigador y las personas de la comunidad o el grupo han de ser horizontales y dialógicas. 
También considera que todas las comunidades disponen de los recursos necesarios para su evolución y transformación. Podría decirse, como lo señala Bernal (2000), para el método de investigación acción participativa, "la teoría y el proceso de conocimiento son, esencialmente, una interacción y transformación recíproca de la concepción del mundo, del ser humano y, por ende, de la realidad". Cabe mencionar que algunos seguidores de este método consideran conveniente utilizar también técnicas cuantitativas, no sólo cualitativas, según lo dicte el transcurso de la investigación.

Grounded theory. La idea básica de este método es que el enfoque positivista es eficaz para someter a prueba teorías hechas, pero impone trabas al desarrollo de nuevas teorías y al logro de descubrimientos mediante la investigación. Asimismo, los defensores de este método cualitativo consideran que es posible elaborar teorías de un menor rango de alcance, es decir más específicas sobre algunos aspectos y patrones particulares de los fenómenos estudiados que se soslayan en el enfoque positivista, pues éste se ocupa de teorías de carácter más general. Por lo anterior, el método de la grounded theory trata de construir una teoría sobre patrones en el fenómeno estudiado mediante la interpretación de datos obtenidos mediante notas de campo sobre la interacción y conversaciones con los miembros del grupo social de interés.

\section{Los métodos cualitativos de investigación vistos desde la Administración}

El análisis de la investigación de Cassel et al (2005) es importante, pues permite avizorar una panorámica general de la investigación cualitativa en Administración desde la perspectiva de varios grupos de interés por su relación con dicha investigación. Cassel, Buehring, Symon, Johnson y Bishop efectuaron 45 entrevistas a profundidad a diferentes grupos de Estados Unidos, Reino Unido y otros países europeos. Entre los entrevistados se encuentran editores de revistas especializadas en la difusión de resultados de investigación en Administración, directivos de asociaciones profesionales vinculadas al ejercicio de la Administración, representantes de organismos patrocinadores de investigación cualitativa, usuarios dentro de las empresas y del gobierno, consultores, practicantes en varios niveles jerárquicos, directores de programas doctorales en Administración y académicos que siguen el camino de la investigación cualitativa, algunos de los cuales han publicado trabajos en revistas de investigación sobre Administración.

Algunos entrevistados consideran que la investigación cualitativa está orientada al logro de la comprensión de cómo se construyen los significados por la interacción social, implicando que el investigador adopte una perspectiva de interpretación en la que el interés fundamental es investigar cuáles son las perspectivas de los sujetos bajo estudio en relación con el contexto que les rodea, incluyendo tanto al entorno inmediato del puesto de trabajo como al nivel más general que abarca a toda la organización y, en última instancia, de la sociedad de la que forman parte. Es decir, opinan que lo que el investigador intenta interpretar mediante los métodos de la investigación cualitativa es la visión del mundo de los sujetos estudiados, siendo importantes estos métodos pues permiten alcanzar un nivel micro en el análisis: el individuo.

También se identifica la investigación cualitativa prácticamente con todo tipo de presentación narrativa que describe los resultados de los estudios realizados en torno a ciertos fenómenos de interés. Algunos opinan que a pesar de que el estilo de presentación de los resultados de la investigación cualitativa se caracteriza por ser eminentemente narrativo y, por tanto de un nivel sólo descriptivo, ese tipo de investigación debería ser profundamente reflexiva. Desde este punto de vista, algunos entrevistados consideran que el investigador orientado por un enfoque cualitativo debe tratar de reflejar las experiencias de los sujetos de estudio, así como las interpretaciones que éstos hacen de la situación que se estudia. La actitud reflexiva que sugieren los entrevistados como algo importante para los investigadores en Administración abarca dos 
aspectos, estrechamente vinculados con la objetividad con que debe realizarse el análisis. El primero de esos aspectos es la reflexión crítica respecto al impacto que tiene en la investigación el propio investigador, es decir, cómo influye su participación en los resultados obtenidos. Por ejemplo, el investigador debería cuestionarse si su papel de entrevistador influyó en el entrevistado y cómo podría haber afectado esa influencia a los resultados de la investigación. El segundo aspecto tiene que ver con el análisis e interpretación de los resultados. Según los entrevistados, el investigador debe analizar críticamente la interpretación que hace de sus experiencias durante la investigación, sea en entrevistas u observaciones realizadas. Miembros de la comunidad académica entrevistados piensan que la actitud reflexiva del investigador es fundamental para representar adecuada y auténticamente las experiencias de los sujetos de estudio.

Hay entrevistados que consideran a la investigación cualitativa como un conjunto de herramientas adecuadas para la investigación en Administración preferible a los métodos cuantitativos. Entre quienes opinan de esta forma, algunos creen que los métodos cualitativos permiten una visión más profunda de la que puede lograrse con métodos cuantitativos, pues permiten ir más allá de los hechos que se observan de manera inmediata de forma tal que se puede obtener una perspectiva más amplia. Algunos van más allá y afirman que los métodos cualitativos pueden usarse para generar teorías, en particular de gran necesidad si el objetivo es realmente entender a las personas y su comportamiento, construyendo esas teorías al ir del nivel de los individuos (nivel micro) hasta el nivel macro. También varios de los entrevistados consideran que la investigación cualitativa consiste en un conjunto de técnicas específicas para reunir información, sin diferenciarlos de los métodos cualitativos. Entre las técnicas que ese grupo identificó con la investigación cualitativa más frecuentemente se encuentran las entrevistas a profundidad, los grupos focales y las entrevista semi estructuradas y no estructuradas.

Para algunos entrevistados la ventaja distintiva de los métodos cualitativos aplicados a la investigación en Administración es que intentan comprender la complejidad de las situaciones; característica típica del entorno actual de las organizaciones y de quienes practican la Administración en ellas, por lo que opinan que esos métodos son más útiles que los métodos cuantitativos. Por ejemplo, casi unánimemente, los investigadores académicos entrevistados concuerdan en que los métodos cualitativos pueden ser más benéficos en la investigación en Administración que los métodos cuantitativos, pues permitirían al investigador describir ampliamente procesos complejos como la toma de decisiones (en la práctica administrativa función de importancia capital y muy compleja). Al respecto creen que los métodos cualitativos pueden contribuir a describir mejor como se toman las decisiones, pues ayudan a comprender la visión que los tomadores de la decisión tienen del mundo que enfrentan.

Sin embargo, no todos los entrevistados consideran que los métodos cualitativos sean preferibles a los métodos cuantitativos. Algunos opinan que la investigación cualitativa puede ser útil para ciertas preguntas de investigación, mas no para todas. En última instancia, consideran, es la naturaleza de la pregunta de investigación la que debe determinar la selección del método de investigación a emplear, no existiendo por lo tanto a priori alguno que pueda considerarse como el correcto. En general, quienes comparten esa posición son entrevistados que abogan también por la posibilidad de la combinación de métodos cualitativos y métodos cuantitativos, ya que aducen que no existe necesariamente un conflicto filosófico fundamental que los haga mutuamente excluyentes sino que, por lo contrario, pueden complementarse. Opinan que los principales beneficios que pueden obtenerse al combinar ambos tipos de métodos es 
mejorar potencialmente la credibilidad de una investigación al proporcionar la posibilidad de comparar los resultados y diseñar mecanismos de supervisión del desarrollo de la investigación.

No obstante, aunque de manera general hay un consenso en la utilidad de combinar ambos métodos entre quienes lo consideran provechoso, existen también matices en su opinión sobre el papel de cada uno de los tipos de investigación. Algunos consideran que el empleo de los métodos cualitativos en la investigación en Administración puede resultar favorable cuando se pretende aclarar resultados que se han obtenido previamente mediante una investigación también cualitativa, así como para profundizar en el análisis de dichos resultados. Otros entrevistados afines a la combinación de métodos sugieren que debido a que la investigación cualitativa es sólo de carácter exploratorio, el uso de los métodos cualitativos debe constreñirse a las etapas iniciales de la investigación. Este segundo grupo opina de esa forma pues consideran que la utilidad principal del uso de los métodos cualitativos es sugerir preguntas e hipótesis de investigación, es decir, piensan que la principal contribución que pueden hacer los métodos cualitativos a la investigación es apoyar al diseño de los estudios.

Para la mayoría de los entrevistados, la investigación cualitativa sufre de falta de credibilidad pues en un entorno caracterizado por el énfasis en los resultados de tipo cuantitativo, como es el caso del ambiente típico en que se desenvuelven los administradores, es un tanto difícil que los resultados descriptivos, típicos de la investigación cualitativa, resulten de gran interés. En el extremo, en la opinión drástica de algunos entrevistados, si no hay algún tipo de medición los datos no pueden transformarse en algo que pueda considerarse como fruto de una investigación, permaneciendo únicamente como anécdotas que incluso siendo interesantes carecen absolutamente de mayor importancia.

Al respecto es conveniente señalar que también hay entre los entrevistados un grupo escéptico sobre la utilidad de los métodos cualitativos en la investigación en Administración. En relación con este punto, es importante destacar que entre los entrevistados que mostraron alguna preocupación por la credibilidad de los métodos cualitativos, existe la creencia de que la resistencia a aceptarlos como adecuados para la investigación en Administración se debe a que no sirven como evidencias fuertes para hacer afirmaciones sobre los objetos de estudio. Uno de los entrevistados percibe a la investigación cualitativa como una recolección de anécdotas carentes de interés para la construcción del conocimiento pues, en última instancia, no prueba punto alguno y siempre es posible encontrar una nueva anécdota que contradiga a todas las anteriores. Otros opinan que basándose en pequeñas muestras los investigadores no pueden presentar argumentos decisivos ni generalizar sus resultados a otras situaciones.

Al parecer, la opinión negativa respecto a la credibilidad de los resultados de la aplicación de los métodos cualitativos en la investigación en Administración no está relacionada únicamente con la falta de cuantificación o de inferencia basada en técnicas estadísticas, ni que se considere a la investigación cualitativa como productora de anécdotas de escaso interés general. También puede jugar un papel importante en esa opinión desfavorable la desconfianza sobre la carencia de espíritu autocrítico del investigador al llevar a cabo su investigación. Dicha desconfianza puede explicar al menos en parte esa opinión, pues si bien algunos entrevistados coinciden en que pueden ser creíbles las conclusiones que presenta un investigador valiéndose de métodos cualitativos, señalan que la credibilidad será posible siempre y cuando dichas conclusiones sean lógicas y el investigador demuestre, sin lugar a dudas, que han sido derivadas como producto de un trabajo metódico, riguroso y objetivo. 
Varios de los entrevistados opinan que los reportes de investigación cualitativa, por lo general, exhiben una gran riqueza de lenguaje. Para algunos esa riqueza, quizá buena para fomentar la comprensión entre los lectores, representa en realidad un peligro. Argumentan que el investigador podría usar lenguaje florido como artilugio para enmascarar debilidades y deficiencias de su investigación como interpretaciones mal fundadas o no objetivas, dando lugar a representaciones equívocas del proceso o situación que el investigador dice haber estudiado a profundidad $y$, en última instancia, tergiversando lo que los sujetos estudiados piensan o sienten en realidad. En una posición extrema, se encuentran entrevistados que consideran que los investigadores que emplean exclusivamente métodos cualitativos en sus estudios lo hacen únicamente como un recurso para evadir el rigor con que tienen que efectuar una investigación. Entre las posibles causas del no empleo de métodos cuantitativos se citaron la falta de preparación de los investigadores y la falta de voluntad para realizar los esfuerzos requeridos por una investigación más rigurosa.

Salvo los totalmente escépticos, la mayoría de los entrevistados coincide en que la formación de doctores en administración debe incluir la preparación para la investigación cualitativa como para la cuantitativa. Sin embargo, no todos coinciden en la importancia relativa de la investigación cualitativa pues mientras unos consideran que la formación en ambos tipos de investigación debe ser de la misma profundidad, otros creen que la formación en investigación cualitativa debe darse como complemento.

\section{Reflexiones y comentarios finales}

En Administración, los trabajos cuya finalidad es analizar el comportamiento y desempeño de las organizaciones comprenden por una parte a los estudios descriptivos y casuísticos de la investigación cualitativa, enmarcados en el dualismo metodológico, y que pueden considerarse dentro de lo que correspondería en las ciencias naturales a una etapa pre-científica de la investigación; y por otra parte, a los estudios explicativos generalizables y replicables de la investigación cuantitativa, que quedan enmarcados dentro del monismo metodológico, y que corresponderían en la ciencia natural a la investigación científica. Finalmente, los estudios propositivos, cuya finalidad es generar herramientas para ser aplicadas en la práctica profesional de conducción de las organizaciones, representarían la generación de tecnologías.

Los métodos cualitativos que se utilizan en la investigación en las disciplinas sociales son muy variados, obedeciendo esa variedad en parte a sus orígenes heterogéneos; y basta un primer acercamiento para percatarse de que se trata de un conjunto de gran magnitud. Asimismo, las diversas fuentes de las cuales han surgido los métodos de la investigación cualitativa hacen que resulte muy complicado ofrecer una descripción completa de ellos. La confusión entre métodos y técnicas también contribuye a complicar más dicha descripción.

Como se ha visto en este ensayo, los métodos cualitativos se han desarrollado supuestamente con el objetivo de ampliar la riqueza de la investigación en diferentes disciplinas sociales. En particular han surgido como intentos para superar supuestas limitaciones de la investigación basada en métodos cuantitativos guiados por el enfoque positivista. Un argumento de quienes defienden esos métodos es que supuestamente cuentan con capacidad para abordar el estudio de la complejidad presente en todos los fenómenos y situaciones de interés para las disciplinas sociales. Se cree que la aceptación y el desarrollo de esos métodos se deben a que permiten que el investigador pueda abordar de manera profunda el estudio de las situaciones que le son de interés. 
Desde muy temprano, la investigación en Administración se ha beneficiado también de los métodos cualitativos. Como ejemplos del empleo de esos métodos que se ha hecho en este campo se encuentran las entrevistas realizadas por Elton Mayo y sus colaboradores, así como la investigación basada en observaciones efectuada por Henry Mintzberg. Incluso, dada la complejidad que en el presente enfrentan las organizaciones y a lo cambiante de su entorno, se puede decir que existe una predisposición natural al empleo de los métodos cualitativos en la investigación en Administración. No obstante, la investigación llevada a cabo por Cassel, Buehring, Symon, Johnson y Bishop (2005), pone de manifiesto que entre diversos tipos de personas involucradas con el desarrollo de la investigación en Administración no existe consenso aún sobre qué es la investigación cualitativa ni sobre varios aspectos relacionados con los métodos propios de ese tipo de investigación en Administración.

Es destacable que no todos los interesados en los métodos cualitativos aplicados a la investigación en Administración comparten el mismo significado. Algunos la definen en los términos generalmente aceptados por los estudiosos de las disciplinas sociales, mientras que otros únicamente consideran que la investigación cualitativa en Administración es toda aquella que no emplea herramientas de medición, tales como el instrumental estadístico. Asimismo, se observa que entre los entrevistados existe la confusión derivada de considerar, erróneamente como se ha señalado al principio de este ensayo, que los métodos y técnicas de investigación significan lo mismo. Para evitar esa confusión, resultaría muy conveniente que los autores discriminaran correctamente entre los métodos y las técnicas de la investigación cualitativa.

Por otra parte, los resultados de la investigación efectuada por Cassel et al (2005), muestran claramente que no existe un consenso general sobre la utilidad de los métodos cualitativos en la investigación en Administración. Algunos rechazan plenamente que dichos métodos y los resultados que éstos producen puedan tener alguna utilidad, en tanto otros consideran que su uso puede ser mejor que el empleo de métodos cuantitativos. Otra posición, que podemos considerar intermedia, sostiene que ambos tipos de métodos pueden ser útiles para enriquecer a la investigación en Administración.

Naturalmente que las posiciones mostradas en el estudio de Cassel et al (2005) respecto a la utilidad de los métodos cualitativos para la investigación en Administración, pueden representar únicamente puntos de vista estrictamente personales, derivados de las preferencias por uno u otro tipo de investigación. Sin embargo, constituyen indicios de cómo se concibe el empleo de esos métodos en ese campo de investigación. Asimismo, los resultados de ese estudio también pueden sugerir algunas ideas para quienes se interesan en la investigación en Administración mediante la aplicación de métodos cualitativos. Para finalizar este ensayo, se enuncian algunas de las sugerencias que podrían derivarse.

Evidentemente, la principal sería que el investigador opte por el empleo de métodos cualitativos para llevar a cabo un estudio, no de manera arbitraria, sino únicamente cuando el problema analizado así lo exija. Sin embargo, es muy difícil señalar con toda precisión en cuáles casos el único recurso para avanzar en la investigación es el uso de tales métodos. Lo que se podría decir en todo caso es que el conocimiento del problema, la existencia de teoría que pueda dar luces al respecto, así como la experiencia del investigador, serían los parámetros a considerar para tomar tal decisión.

También podría ser conveniente para avanzar en la investigación en Administración el seguir estrategias de integración de la investigación con métodos cualitativos con la realizada mediante métodos cuantitativos. Respecto a la decisión de cuál o cuáles estrategias de integración deberían seguirse podrían considerarse los mismos parámetros mencionados en el párrafo previo. Quizá podría sugerirse que el investigador optase en 
todos los casos que fuese posible por llevar a cabo, al menos, la estrategia de triangulación y, mejor aún, desde la perspectiva de la triangulación múltiple.

No debe perderse de vista la importancia que tiene la actitud reflexiva en la investigación basada en métodos cualitativos, así como al reportar resultados. Aunque la reflexión no es exclusiva de ese tipo de investigación, pues también es esencial en la investigación con métodos cuantitativos, la valía de los resultados obtenidos por el investigador depende en gran medida de que se alcancen garantizando que se han hecho los mejores esfuerzos por preservar la esencia de las características del caso o casos estudiados. La actitud reflexiva es de gran importancia, pues el principal criterio para juzgar los resultados de la investigación cualitativa es que sean creíbles. Sin embargo, el investigador que emplea métodos cualitativos, en general en la investigación en las diversas disciplinas sociales y en particular en la investigación en Administración, debe estar consciente que dicho criterio podría ser un tanto débil. A final de cuentas decir que algo es creíble no significa exactamente lo mismo que decir que es verdadero. Precisamente el que ese criterio sea el comúnmente aceptado para evaluar los resultados de la investigación cualitativa constituye su principal debilidad.

\section{Nota}

(1) Una primera versión de este ensayo aparece en la memoria del XIII Congreso de la Academia de Ciencias de la Administración (México, D. F., julio de 2009).

\section{Bibliografía}

Alonso, J. A. 1996. Metodología. México: Limusa.

Bericat, E. 1998. La integración de los métodos cuantitativos y cualitativos en la investigación social. Barcelona: Ariel.

Berkeley, G. 1910. A new theory of vision and other writings. London: J. M. Dent \& Sons Ltd.

Bernal, C. 2000. Metodología de la investigación para administración y economía. Santa Fé de Bogotá: Pearson.

Cassel, C; Buehring, A; Symon, G; Johnson, P. y Bishop, V. 2005. Qualitative management research: a thematic analysis of interviews with stakeholders in the field. Benchmarking good practices in qualitative management research: Final report. Research methods programme. London: Economic and Social Research Council.

Cereceres, L. 2003. Aplicación del estudio de caso en el análisis organizacional. Ponencia. Primer Congreso Internacional de Análisis Organizacional. México, Iztapalapa: 3-5 diciembre.

Ferrarotti, F. 1993. Sobre la autonomía del método biográfico. En: Marinas, M y Santamaría, C. La historia oral: Métodos y experiencias. Madrid: Edit. Debate.

Fetterman, D.M. 1989. Ethnography step by step. California: Sage.

Gephart, R. P. 1999. Paradigms and research methods. Research Methods Forum 4. Academy of Management, Research Methods Division, Texas University. http://bit.ly/14uqYS 
Gephart, R.P. 1993. The textual approach: risk and blame in disaster sensemaking. Academy of Management Journal 36(6): 1465-1514.

Gómez, L. 2004. Evolución de variables financieras a lo largo del ciclo de vida. Tesis doctoral no publicada. México: DCA, UNAM.

González, F. L. 2000. Investigación cualitativa en psicología. México: Thompson.

Grawitz, M. 1974. Méthodes des sciences sociales. Paris: Dalbor.

Guba, E.G. y Lincoln, Y.S. 1994. Competing paradigms in qualitative research. En: N.K. Denzin y Y.S. Lincoln (Comps) Handbook of qualitative research. Newbury Park, CA: 105-117.

Hancock, B. 2002. An introduction to qualitative research. Nottingham: University of Nottingham.

Hill, C. y Jones, G. 1995. Strategic management: an integrated approach. Boston: Houghton Mifflin Co.

Jick, T. 1993. Managing change: cases and concepts. Illinois: Richard D. Irwin, Inc.

Kolakowski, L. 1981. La filosofía positivista. Desde Hume hasta el Círculo de Viena. Madrid: Cátedra.

Lussier, R. 1997. Management: concepts, applications and skill development. Springfield, MA: SouthWestern College Publishing.

Martínez, M. 1999. La nueva ciencia: Su desafío, lógica y método. México: Trillas.

Merino, G. 1985. Economías de escala en el sistema bancario mexicano. Tesis profesional no publicada, ITAM.

Ordóñez, J. 2003. Ciencia, tecnología e historia. Colección Cuadernos de la Cátedra Alfonso Reyes. México: FCE-ITESM.

Pedone, C. 2000. El trabajo de campo y los métodos cualitativos. Necesidad de nuevas reflexiones desde las geografías latinoamericanas. Scripta Nova 4(57). http://www.ub.es/geocrit/sn-57.htm

Philip, L.J. 1998. Combining quantitative and qualitative approaches to social research in human geography, an impossible mixture? Environment and planning A 30(2): 261-276.

Pita, S. y Pértegas, S. 2002. Investigación cuantitativa y cualitativa. Nota técnica. Coruña: Unidad de Epidemiología Clínica y Bioestadística. Complexo Hospitalario-Universitario Juan Canalejo España.

Ragin, C. C; Nagel, J. y White, P. 2004. The Workshop on Scientific Foundations of Qualitative Research. Reporte. Virginia: National Science Foundation. http://bit.ly/3XEND3

Rendón, M. y Montaño, L. 2004. Las aproximaciones organizacionales. Caracterización, objeto y problemática. Contaduría y Administración 213: 101-120.

Rumelt, R. 1986. Strategy, structure and economic performance. Boston: Harvard Business School Press. 
Saavedra, M. 2002. La valuación de empresas: enfoques teóricos y aplicación de los modelos Black y Scholes, Valor económico agregado y Flujo de efectivo disponible en México 1991-2000. Tesis doctoral no publicada. México: DCA, UNAM.

Schwandt, T. 1994. Constructivist, interpretivist approaches to human inquiry. En: N. Denzin y Y. Lincoln (eds.) Handbook of qualitative research. Thousand Oaks: Sage Publications, pp. 118-37.

Schwartzman, H.B. 1993. Ethnography in organizations. California: Sage.

Scribano, A. 2000. Reflexiones epistemológicas sobre la investigación cualitativa en disciplinas sociales. Cinta moebio 8: 1-20. http://www.moebio.uchile.cl/08/scribano.htm

Stake, R.E. 1999. Investigación con estudio de casos. Madrid: Morata.

Taylor, S.J. y Bogdan, R. 1987. Introducción a los métodos cualitativos de investigación. Madrid: Paidós.

Valles, M. 1997. Técnicas cualitativas de investigación social. Reflexión metodológica y práctica profesional. Madrid: Síntesis.

Vera, A. 2005. Diálogo entre lo cuantitativo y lo cualitativo en la investigación científica. El desafío de la triangulación. Disciplina y trabajo 7(15): 38-40.

Vera, A. y Villalón, M. 2005. La triangulación entre métodos cuantitativos y cualitativos en el proceso de investigación. Disciplina y trabajo 7(16): 85-87.

Waters, C. K. 1998. Causal regularities in the biological world of contingent distributions. Biology and philosophy 13: 5-36.

Recibido el 20 Jul 2009

Aceptado el 5 Sep 2009 